\title{
MICROWAVE-INDUCED THERMOACOUSTIC IMAGING FOR EARLY BREAST CANCER DETECTION
}

\author{
ZHONG JI, YONG FU and SIHUA YANG* \\ MOE Key Laboratory of Laser Life Science and Institute \\ of Laser Life Science, South China Normal University \\ Guangzhou 510631, P. R. China \\ *yangsh@scnu.edu.cn
}

Received 29 October 2012

Accepted 15 November 2012

Published 31 January 2013

\begin{abstract}
Microwave-induced thermoacoustic tomography (TAT) is a noninvasive, nonionizing modality based on the inherent differences in microwave absorption of malignant breast tissues and normal adipose-dominated breast tissues. In this paper, a TAT system based on multielement acquisition system was built to receive signals. Slices from different layers in the sample were composed into a three-dimensional (3D) volume. Based on the 3D volume, inherent differences in microwave absorption between different biological tissues can be converted into structure information. Our experimental results of some mimicked and human tumors indicate that TAT may potentially be used to detect early-stage breast cancers with high contrast.
\end{abstract}

Keywords: Early breast cancer; microwave-induced thermoacoustic tomography; dielectric properties.

\section{Introduction}

Breast cancer, which is a leading cause of cancer deaths among women, can be much improved by detection at its early stage. X-ray mammography, ultrasonography, and nuclear magnetic resonance imaging (NMRI) are the current clinical tools for detection of breast cancer. ${ }^{1-3}$ However, mammography inevitably brings about exposure to ionizing radiation and has difficulty in imaging pre-menopausal breasts. Ultrasonography, which usually used as an adjunct tool to X-ray mammography, tends to miss nonpalpable tumors and has poor imaging contrast. NMRI is suitable to detect tumors that usually have high water content. However, limited by its long detection time and high price, there is a tendency to not use NMRI in a large-scale physical examination. For this reason, mammography, ultrasonography, and NMRI are unsuited to medical census to detect early breast cancer.

*Corresponding author.

This is an Open Access article published by World Scientific Publishing Company. It is distributed under the terms of the Creative Commons Attribution 3.0 License. Further distribution of this work is permitted, provided the original work is properly cited. 
Microwave-induced thermoacoustic tomography (TAT), which is similar to photoacoustic imaging, is a hybrid of microwave imaging and ultrasound imaging, as it achieves excellent microwave absorption contrast and ultrasound spatial resolution. ${ }^{4}$ As the thermoacoustic (TA) wave contains microwave absorption characteristics of the tissue, TAT can map the microwave absorption distribution, which is related to the physiological state. Therefore, the detection of breast tumor by TAT is feasible, and recently, increasing attention is paid to applying TAT to the detection of breast cancer. ${ }^{5-10}$

In this paper, we developed a TAT system and performed experiments to demonstrate the feasibility of detecting and evaluating early breast tumor. The results indicate that the nonionizing TAT may contribute to the detection and estimation of early-stage breast cancer and has the potential to be used for general examination of early breast cancer.

\section{Approach and Methods}

\subsection{Theory of thermoacoustic imaging}

When a biological tissue is illuminated by a microwave pulse, the electromagnetic energy changes into heat. A small transient temperature rise can be produced, the heated tissue then expands and contracts, and the thermoacoustic wave emerges as the output. This thermoacoustic wave $p(r, t)$ can be expressed by the following equation ${ }^{11}$ :

$$
p(r, t)=\frac{\beta I_{0} c_{0}}{4 \pi C_{p}} \tau \frac{d}{d t} \iint_{\left|r-r^{\prime}\right|=c_{0} t} A\left(r^{\prime}\right) \frac{d r^{\prime}}{c_{0} t},
$$

where $\beta$ is the isobaric volume expansion coefficient, $I_{0}$ is intensity of the irradiating energy (assumed to be constant throughout the tissue volume), $c_{0}$ is the speed of ultrasound, $C_{p}$ is the specific heat, and $A\left(r^{\prime}\right)$ is the spatial distribution of microwave absorption coefficient at position $r^{\prime}$. From Eq. (1), TAT can map the spatial distribution of microwave absorption coefficients, because $\beta, c_{0}$, and $C_{p}$ vary little between different tissues.

\subsection{Thermoacoustic computed tomography}

In this paper, a combined scanning technology was used to realize 3D imaging, as shown in Fig. 1. Multielement linear focus detector in the X-Y plane

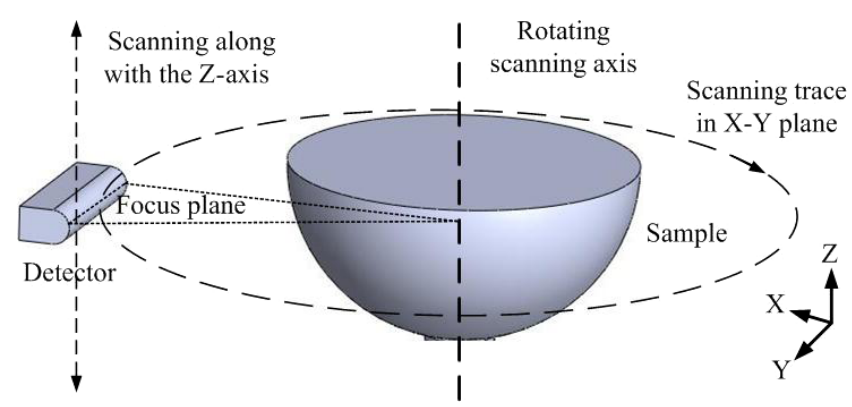

Fig. 1. Schematic of three-dimensional thermoacoustic scanning.

was along the center of the object to set a rotating scanning. Usually, once signals are gathered from the transducer at a 100 positions, the filterback projection method can effectively reconstruct a twodimensional image which reflects the microwave absorption differences. After acquisition of a twodimensional image, the detector is placed along the $Z$-axis for scanning. Slices from different layers in the sample were composed into a $3 \mathrm{D}$ volume.

\subsection{Microwave absorption coefficient of breast tissues}

According to electrodynamics, the microwave absorption coefficient of biological tissues can be expressed as:

$$
\alpha=\omega \sqrt{\mu \varepsilon}\left[\frac{1}{2}\left(\sqrt{1+\frac{\sigma^{2}}{\varepsilon^{2} \omega^{2}}}-1\right)\right]^{\frac{1}{2}},
$$

where $\omega$ is the angular frequency of microwave irradiated, $\mu$ is the permeability; $\varepsilon$ is the relative dielectric constant, and $\sigma$ and is the conductivity. Usually, the relative dielectric constant and conductivity are taken into account because permeability is of little difference in the biological tissues. At $6 \mathrm{GHz}$, the relative dielectric constant and conductivity of the tumor tissue is 10 times bigger than the normal breast tissue. ${ }^{12}$ The microwave absorption coefficient was calculated by Eq. (2), shown in Fig. 2, which indicates that the microwave absorption coefficient of malignant breast tissues is 45 to $90 \mathrm{~m}^{-1}$ larger than the microwave absorption of normal adipose-dominated breast tissues. The difference in microwave absorption coefficient between the malignant and normal breast tissues is large enough for detection by TAT. It is also demonstrated that the TAT can reveal 


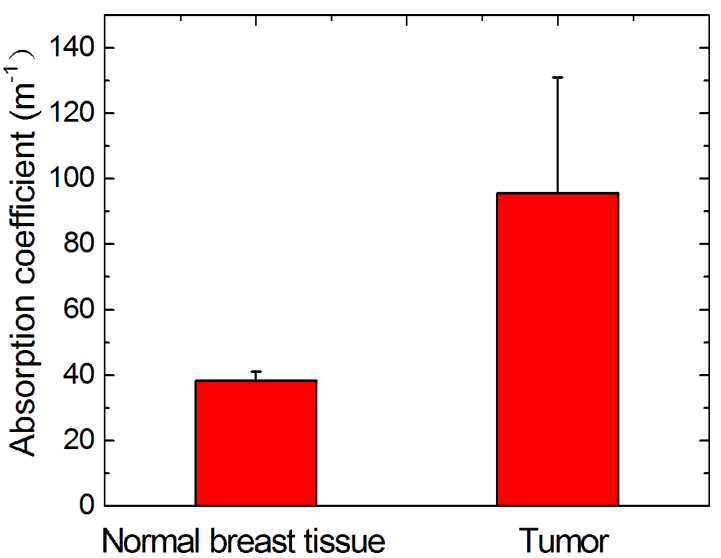

Fig. 2. Microwave absorption coefficient of normal breast tissue and tumor.

label-free margin morphological features of tumors based on intrinsic contrast.

\subsection{Data acquisition system and imaging procedure}

The schematic of thermoacoustic system is shown in Fig. 3, and a Cartesian coordinate system $(X, Y, Z)$ is also depicted. A $6 \mathrm{GH}$ microwave generator (BW-6000HPT, China) transmitted $0.5 \mu \mathrm{s}$ microwave pulses at a maximum pulse repetition frequency of $500 \mathrm{~Hz}$ controlled by a function generator. The size of waveguide for microwave radiation is $34.8 \times 15.8 \mathrm{~mm}^{2}$.

The sample was immersed in a polyvinyl chloride tank and fixed on a sample stage which can rotate and lift to achieve 3D scanning. Both sample and stage were immersed in mineral oil to couple thermoacoustic waves.
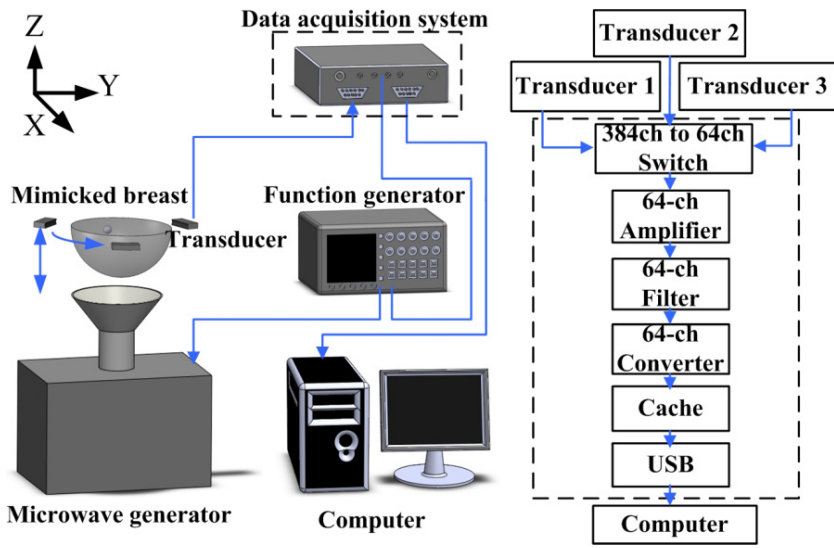

Fig. 3. Schematic of the experimental setup for microwaveinduced thermoacoustic data acquisition system.
Three commercial linear transducer arrays (L2L50A, SIUI, China) are used, which are similar to the transducer used in our previous study, ${ }^{13,14}$ with 128 elements arranged at the three vertices of the equilateral triangle. The central frequency of the linear multielements transducer array was $2.5 \mathrm{MHz}$ with a bandwidth of $70 \%$.

A custom-made parallel data acquisition system (DAS) was employed to transmit the acquired raw thermoacoustic signals from the transducer arrays. The signals from each transducer array are acquired at the same time. After time-division multiplexing circuit, the 384-channel $(384 \mathrm{ch})$ signals came from three transducers are converted into six groups, and signals of each group (64 ch) are amplified, filtered, and acquired in proper sequence. Then the signals of six groups are recorded in the computer in order. The DAS is triggered by the same function generator which triggers the microwave generator synchronously. With the parallel DAS, a frame of signals is acquired and transmitted in $1 / 100 \mathrm{~s}$. To acquire $2 \mathrm{D}$ image of the sample, the sample stage scans around the axial of center of waveguide for 10 steps, with each step of $36^{\circ}$, when the three transducers keep relative position unchanged. At each step, signals are averaged by eight times. Therefore, when using microwave repetition frequency of $10 \mathrm{~Hz}$, it takes less than $1 \mathrm{~s}$ for the acquisition, transmission, recording, and mechanical motion for a step and the whole scan process for acquisition of a $2 \mathrm{D}$ image lasts about $10 \mathrm{~s}$. To acquire 3D volume, the steps of $2 \mathrm{D}$ image data acquisition above are repeated along the axis of the center of waveguide for 40 steps, with step distance of $0.5 \mathrm{~mm}$.

The signals acquired from each horizontal plane of the sample were calculated by an image filtered backprojection reconstruction algorithm. By VolView (Kitware, Inc., USA), slices of the sample were composed into a $3 \mathrm{D}$ volume.

\section{Results}

\subsection{D imaging of mimicked samples}

TAT provides a cross-sectional image, and it is necessary to adjust the detecting cross-section carefully to the tumor before the experiment. However, in the actual detecting process, it is impossible to know the tumor deposition in advance and it is likely to miss the tumor if only one crosssection in the breast is detected. Therefore, it is 
necessary to detect multiple cross-sections covering the entire breast and reconstruct a 3D volume. More significantly, the largest diameter and volume of the tumor, which are important parameters to estimate clinical pathological stage of the tumor and decide treatment, can be judged from the 3D volume.

In this paper, a subuniform mimicked tumor, whose height is about $18 \mathrm{~mm}$, was used to test the 3D imaging capability of this system, shown in Fig. 4. In Fig. 4(a), the reconstructed 3D volume can be seen, and actual sample in size and relative position are highly consistent as well. Figure 4(b) shows the four slices of the $3 \mathrm{D}$ volume, which is $2 \mathrm{~mm}$ apart from its upper slice. The diameter of the highlighted areas indicates the difference of microwave absorption coefficient and has obvious difference between the various slices; for each image, contrast was enough to distinguish the image anomaly area.

The microwave-induced thermoacoustic waves emitted from the boundaries and notches of the round are blocked in virtue of microwave absorption heterogeneity. However, subjected to spatial resolution $^{15}(0.5 \mathrm{~mm})$, signals from boundary of forward and backward cannot be distinguished if the diameter of tissue is less than or equals to $1 \mathrm{~mm}$. Therefore, the reconstructed image of tissue whose diameter less than or equals to $1 \mathrm{~mm}$ will be a solid point, and the reconstructed image of tissue whose diameter is more than $1 \mathrm{~mm}$ will be a circular ring, which is a good coincidence with the result in Fig. 4. Therefore, the $3 \mathrm{D}$ image obtained by this system is reliable.

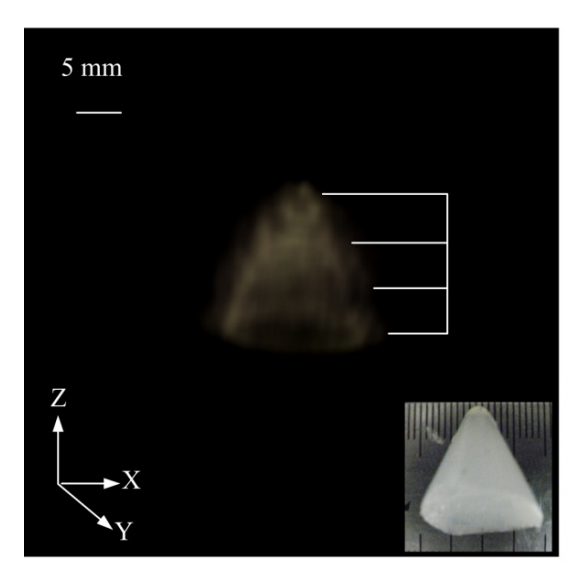

(a)

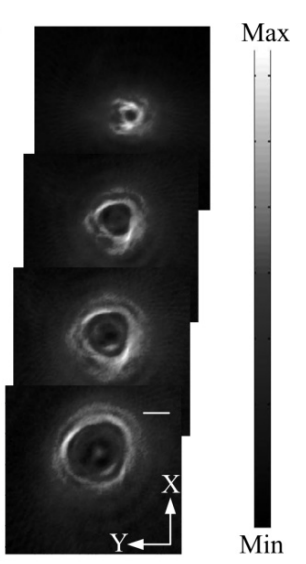

(b)
Fig. 4. (a) Three-dimensional thermoacoustic imaging of a mimicked tumor. (b) Four slices of two-dimensional thermoacoustic images of the tumor correspond to Fig. 4(a).

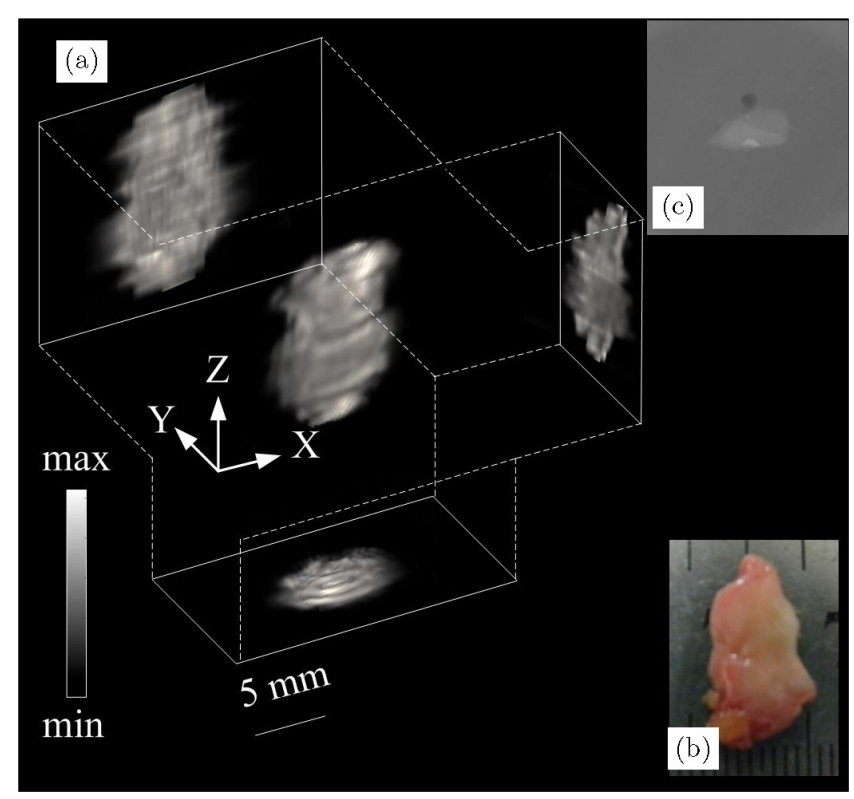

Fig. 5. (a) Three-dimensional thermoacoustic imaging of a human breast tumor. (b) Photographs of breast tumor after surgery. (c) X-ray image of breast tumor in top view.

\subsection{D imaging of human tumor}

A 3D imaging experiment of a human tumor, which was acquired from the First Affiliated Hospital of Ji'nan University, China, was conducted. The tumor was embedded $2 \mathrm{~cm}$ into the fat, which is part of a simulated normal breast tissue. Figure 5(a) depicts a $3 \mathrm{D}$ reconstructed TAT volume and the maximum amplitude projections in $\mathrm{XZ}, \mathrm{YZ}$ and $\mathrm{XY}$ planes. It can be seen that the maximum amplitude projections of XY-plane well corresponded with the $\mathrm{X}$-ray image of the top view in Fig. 5(c). Figure 5(b) shows the photograph of the tumor after surgical extraction.

Through the three-dimensional imaging, changes of electromagnetic parameters, which indicate pathological changed, can be changed into structure information. Some medical information, such as shape, location and size of the tumor, can be obtained by the $3 \mathrm{D}$ volume.

\section{Discussion}

According to the IEEE standard,${ }^{16}$ when microwave exposure occurs under a controlled environment, which means exposure to persons who are aware of the potential of exposure and use for partialbody exposure, the upper limit of energy deposition 
is $20 \mathrm{~mW} / \mathrm{cm}^{2}$ for $6 \mathrm{GHz}$ microwaves. Microwave pulse energy in our system can be estimated as following: Supposing the microwave energy is uniform after emergence in waveguide $(3.48 \times$ $1.58 \mathrm{~cm})$, the energy deposition in surface of the biological tissue can be estimated by the microwave pulse energy and radiation area. In the experiment, the display of voltage meter in microwave generator is $25 \mathrm{KV}$, which corresponds to the microwave peak power is $80 \mathrm{KW}$, according to the specification of microwave generator. The pulse width is about $0.5 \mu \mathrm{s}$. The energy density is $E \approx(80 \times 0.5) /$ $(3.48 \times 1.58)=7.3 \mathrm{~mJ} / \mathrm{cm}^{2}$ in waveguide, and that the power deposition is $73 \mathrm{~mW} / \mathrm{cm}^{2}$ at repetition frequency of $10 \mathrm{~Hz}$. In fact, the microwave illuminated area will expand sharply due to diffraction after the microwave exits the waveguide. In actual detection, the sample or breast is placed at a distance from the waveguide, where the power density is much less than $73 \mathrm{~mW} / \mathrm{cm}^{2}$ due to space scattering. In this way, the energy density is close to safety standards $20 \mathrm{~mm}$ away from the waveguide, as our repetition frequency is $10 \mathrm{~Hz}$. In the detection process, reducing the microwave peak power and the pulse repetition frequency can ensure safety. In the future, we will use antenna with high directivity to transmit microwave, in order to increase the illuminated area to ensure safety and ensure that the microwave energy covered the whole breast. Also, the space divergence can be reduced, so that the SNR in deep depth can be improved, where SNR $=A_{s} / A_{n}$, with $A_{s}$ being the average amplitude of the microwave signal and $A_{n}$ representing the average amplitude of undesired noise.

Microwave could achieve excellent penetration depth. Theoretically, the penetration depth can be expressed as:

$$
d=1 / \alpha,
$$

where $\alpha$ is the absorption coefficient. Therefore, the penetration depth in normal adipose-dominated breast tissues or in fat with $6 \mathrm{GHz}$ is about $28.5 \mathrm{~mm}$. In fact, it has the possibility of receiving signal when sample is below penetration depth, because $36.8 \%$ of microwave energy remains at the penetration depth. In Fig. 6, the relationship between signal intensity and depth is experimentally measured. The sample is round $5 \%$ agar that is $10 \mathrm{~mm}$ in diameter. The distance between the axis line of the transducer and the waveguide is defined as $d$. The sample, which was set to be at the same altitude as

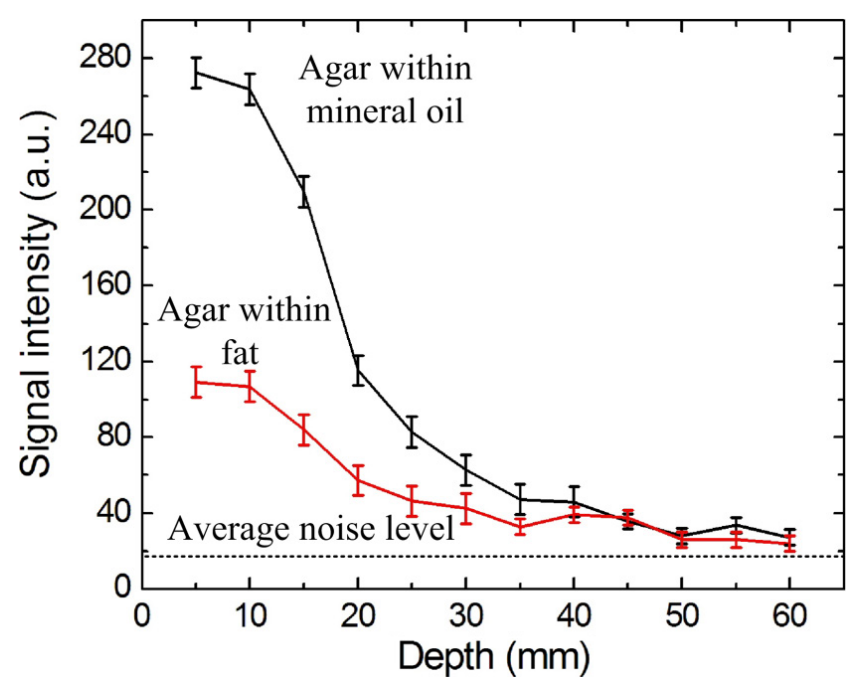

Fig. 6. Relationship between signal intensity and depth in mineral oil and fat.

the transducer, was placed in a container that was filled with fat. At first, $d$ was set to be $5 \mathrm{~mm}$. Then, $d$ was increased to $5 \mathrm{~mm}$, namely, the sample and transducer drops $5 \mathrm{~mm}$ at the same time in each step. This curve reflects the combined action of spatial divergence and the attenuation in fat, with the former playing a leading role. Served as controls, a similar experiment was performed using mineral oil. At the time of the experiment, the whole space is filled with mineral oil, which is a weak microwave absorption substance, so this curve reflects the spatial divergence of the microwave energy. In Fig. 6 , it is clear to see that $\mathrm{SNR}=1.5$ in mineral oil and $\mathrm{SNR}=1.3$ in fat at a depth of $60 \mathrm{~mm}$. The results show that the imaging depth of TAT can achieve $60 \mathrm{~mm}$, which is deeper than the microwave penetration depth. Therefore, TAT can detect tumor at a depth of $60 \mathrm{~mm}$ which is the maximum depth at which breast tumor exists.

\section{Acknowledgments}

This research is supported by the National Basic Research Program of China (2011CB910402; 2010CB732602), the Program for Changjiang Scholars and Innovative Research Team in University (IRT0829), the National Natural Science Foundation of China $(81127004,11104087)$, the Foundation for Distinguished Young Talents in Higher Education of Guangdong, China (LYM10061), the Specialized Research Fund for the Doctoral Program 
of Higher Education (20114407120001), and the Science and Technology Project of Bejing, China (2012J4100114).

\section{References}

1. M. Sabel, H. Aichinger, "Recent developments in breast imaging," Phys. Med. Biol. 41, 315-368 (1996).

2. S. Venkataraman, N. Hines, P. J. Slanetz, "Challenges in mammography: Part 2, Multimodality review of breast augmentation-imaging findings and complications," Amer. J. Roentgenol. 197, W1031W1045 (2011).

3. A. Karellas, S. Vedantham, "Breast cancer imaging: A perspective for the next decade," Med. Phys. 35, 4878-4897 (2008).

4. R. A. Kruger, K. K. Kopecky, A. M. Aisen, D. R. Reinecke, G. A. Kruger, W. L. Kiser, "Thermoacoustic CT with radio waves: A medical imaging paradigm," Radiology 211, 275-278 (1999).

5. R. A. Kruger, K. D. Miller, H. E. Reynolds, W. L. Kiser, D. R. Reinecke, G. A. Kruger, "Breast cancer in vivo: Contrast enhancement with thermoacoustic CT at $434 \mathrm{MHz}$ - Feasibility study," Radiology 216, 279-283 (2000).

6. M. Xu, G. Ku, X. Jin, L. V. Wang, B. D. Fornage, K. K. Hunt, "Breast cancer imaging by microwaveinduced thermoacoustic tomography," Photons Plus Ultrasound: Imaging and Sensing, Vol. 5697, A. A. Oraevsky and L. V. Wang, Eds., pp. 45-48, Spie-Int Society of Optical Engineering, Bellingham (2005).

7. M. Pramanik, G. Ku, C. H. Li, L. V. Wang, "Design and evaluation of a novel breast cancer detection system combining both thermoacoustic (TA) and photoacoustic (PA) tomography," Med. Phys. 35, 2218-2223 (2008).
8. G. R. Zhu, M. Popovic, Q. Q. Fang, "Microwaveinduced thermoacoustics: Assisting microwave tomography," IEEE Trans. Magn. 45, 1654-1657 (2009).

9. L. Nie, D. Xing, Q. Zhou, D. Yang, H. Guo, "Microwave-induced thermoacoustic scanning CT for high-contrast and noninvasive breast cancer imaging," Med. Phys. 35, 4026-4032 (2008).

10. L. Nie, Z. Ou, S. Yang, D. Xing, "Thermoacoustic molecular tomography with magnetic nanoparticle contrast agents for targeted tumor detection," Med. Phys. 37, 4193-4200 (2010).

11. G. Ku, L. V. Wang, "Scanning microwave-induced thermoacoustic tomography: Signal, resolution, and contrast," Med. Phys. 28, 4-10 (2001).

12. M. Lazebnik, D. Popovic, L. McCartney, C. B. Watkins, M. J. Lindstrom, J. Harter, S. Sewall, T. Ogilvie, A. Magliocco, T. M. Breslin, W. Temple, D. Mew, J. H. Booske, M. Okoniewski, S. C. Hagness, "A large-scale study of the ultrawide band microwave dielectric properties of normal, benign and malignant breast tissues obtained from cancer surgeries," Phys. Med. Biol. 52, 6093-6115 (2007).

13. B. Yin, D. Xing, Y. Wang, Y. Zeng, Y. Tan, Q. Chen, "Fast photoacoustic imaging system based on 320-element linear transducer array," Phys. Med. Biol. 49, 1339-1346 (2004).

14. F. Ye, S. Yang, D. Xing, "Three-dimensional photoacoustic imaging system in line confocal mode for breast cancer detection," Appl. Phys. Lett. 97, 213702 (2010).

15. L. Nie, D. Xing, Q. Zhou, D. Yang, H. Guo, "Microwave-induced thermoacoustic scanning CT for high-contrast and noninvasive breast cancer imaging," Med. Phys. 35, 4026-4032 (2008).

16. IEEE Standard for Safety Levels with Respect to Human Exposure to Radio Frequency Electromagnetic Fields $3 \mathrm{kHz}$ to $300 \mathrm{GHz}$, IEEE Standard C95.1, 1999 . 